\title{
不确定浅海环境中基于水平阵稳健子空间估计的 宽容检测方法
}

$$
\text { 王宣 }{ }^{1} \text {, 孙超 }{ }^{1} \text {, 向龙风 }{ }^{2} \text {, 李明杨 }{ }^{1}
$$

(1.西北工业大学 航海学院, 陕西 西安 710072；2. 电子信息控制重点实验室，四川 成都 610036)

\begin{abstract}
摘 要: 环境参数在复杂浅海环境中通常是不确定的,从而制约了匹配模型类高性能方法的检测性 能。对于水下目标检测问题, 需要更宽容的检测方法。在以往的研究中, 稳健模态子空间检测方法具 有兼顾高性能和稳健性的特点, 但该检测器适用于垂直阵, 性能受浅海深度的限制, 因此考虑适用于 水平阵的宽容检测方法。子空间检测方法是一类具有一定稳健性的检测方法, 其性能取决于信号子 空间的估计。通过不确定波数重采样的方式将不确定环境和未知目标位置参数引入水平阵信息观测 矩阵,得到了不确定环境中信号子空间的稳健估计方法。结合信号子空间的稳健估计提出了一种对 不确定参数具有宽容性的子空间检测器, 通过浅海不确定环境中的仿真检验, 证明该检测器具有较高 的平均检测能力同时对不确定参数有一定的宽容性。
\end{abstract}

\section{关 键 词: 检测; 不确定环境;水平阵;子空间}

中图分类号:TB56

文献标志码: A

当海洋环境和目标位置参数精确已知时, 可以 利用水下声传输函数构建最优检测器。但受限于复 杂多变的海洋环境和当前的测量手段, 往往不能获 得正确的参数信息, 这就导致传输函数失配 ${ }^{[1-3]}$, 进 而影响检测器检测性能 ${ }^{[4]}$ 。

针对不确定环境中的检测问题, 常见的宽容检 测器有贝叶斯检测器和广义似然比检测器 ${ }^{[5]}$ 。贝 叶斯检测器通过积分的形式将参数不确定性引人检 测器, Sha 等证明了在不同的参数条件下, 贝叶斯检 测器具有最优的平均检测性能 ${ }^{[6]}$ 。但在实现中, 贝 叶斯检测器性能对参数的采样存在依赖, 在部分未 采样到的参数条件下检测能力明显低于平均检测能 力 $^{[7]}$, 这就导致检测性能不够稳健。广义似然比检 测器性能接近贝叶斯检测器 ${ }^{[8]}$, 同样具有很好的平 均检测性能, 在部分未采样到的参数条件下不够稳 健。能量检测器没有利用先验信息, 不受参数变化 的影响, 具有最稳健的检测性能 ${ }^{[9]}$, 但能量检测器 只利用了信号的能量信息, 对较弱信号的检测能力 较差。近年来, $\mathrm{Li}$ 等结合简正波传播模型和垂直阵
信息观测模型，提出了一种稳健模态子空间检测 器 ${ }^{[7]}$ 。该检测器将不同参数条件下的最大模态子 空间作为信号子空间的稳健估计, 对投影到该子空 间的信号进行能量检测，在保证检测稳健性的前提 下仍有较高的检测能力。但因为模态子空间由模态 函数矩阵张成, 这意味着只有垂直阵接收信号能落 人该子空间中,所以稳健模态子空间检测器只能用 于垂直阵。但垂直阵在浅海中孔径受到海深限制、 应用场景不够灵活, 故垂直阵对于浅海不确定环境 中的宽容检测问题有一定局限。

水平阵不存在孔径受限的问题,通常能带来更 大的阵列增益, 是一种应用场景广泛的常用阵 形 ${ }^{[10-12]}$, 但近些年水平阵的检测相关研究较少, 更 多的研究集中于测向、定位等领域, 因此研究适用于 水平阵的宽容检测方法是很有必要的。在检测方法 的研究中, 首要的问题是建立适当的接收与观测模 型。Bogart 等在研究中给出了波导环境中的水平阵 接收信号的远场近似模型 ${ }^{[11]}$, 在此基础上, Conan 等给出了目标在端射方向时水平阵的远场观测矩阵 
形式 ${ }^{[13]}$, 结合两者的研究, 可以得到水平阵一般远 场接收与观测模型。接着, 在一般接收与观测模型 的基础上, 研究不确定参数条件下的宽容检测方法。 前人的研究指出, 利用观测矩阵构建信号子空 间 ${ }^{[14]}$, 通过将信号向恰当子空间投影的方式可以在 提高检测能力的同时保证一定的稳健性 ${ }^{[7]}$ 。因此, 考虑利用这一思路, 通过水平阵观测矩阵估计信号 子空间, 在此基础上得到宽容的检测方法。但是, 在 实际中, 受风浪、涡流、测量技术等影响, 环境参数和 目标位置往往是未知的, 而水平阵远场观测矩阵在 不同条件下可能是不同的,对应子空间也存在差异。 针对这一问题, 将环境参数和目标位置参数不确定 性引人观测矩阵, 通过不确定波数采样和奇异值分 解的方法, 构建了一个能包含各参数条件对应信号 的稳健信号子空间, 其估计误差可以由相对投影误 差度量。在信号子空间稳健估计的基础上提出一种 水平阵稳健子空间检测器 (horizontal array robust subspace detector, HRSD), HRSD 通过对投影到稳健 信号子空间的信号进行能量检测, 在具有较高的检 测能力的同时对不确定参数具有宽容性。

\section{1 海洋波导中简谐声源的水平阵信息 观测}

在水平不变浅海环境中,建立如图 1 所示三维 坐标系,其中 $x O y$ 平面表示海平面, $z$ 轴表示深度。 一个阵元间距为 $d$ 的 $N$ 元均匀水平线列阵 ( horizontal line array, HLA) 平行 $x$ 轴放置于深度 $z_{\mathrm{s}}$ 处, 各阵元坐标分别为 $\left(0,0, z_{\mathrm{a}}\right), \cdots,((N-1) d, 0$, $\left.z_{\mathrm{a}}\right)$ 。一远场声源 $S$ 深度为 $z_{\mathrm{s}}$, 与 $O$ 点水平距离为 $r_{\mathrm{s}}$, 与 $y$ 轴夹角为 $\theta$ 。

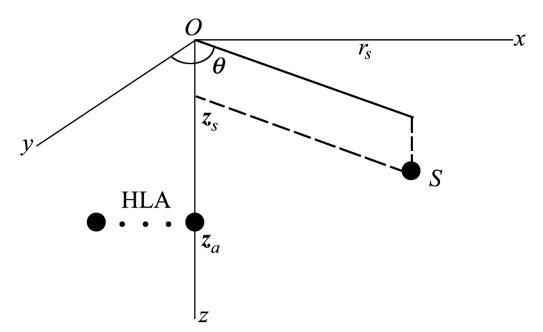

图 1 声源、阵列参数示意图

根据简正波理论 ${ }^{[15]}$, 在水平不变浅海环境中, 辐射声源与远场某一阵元之间的信道传输函数可以
近似写作一系列简正波之和的形式

$$
\begin{gathered}
g_{n}(f, \boldsymbol{S}, \boldsymbol{\Phi})=\sum_{m=1}^{M} \frac{\mathrm{e}^{\mathrm{j} \pi / 4}}{\rho\left(z_{\mathrm{s}}\right)} \sqrt{\frac{2 \pi}{k_{m} r_{\mathrm{s}}}} \Psi_{m}\left(z_{\mathrm{a}}\right) \Psi_{m}\left(z_{\mathrm{s}}\right) \mathrm{e}^{\mathrm{j} k_{m} r_{\mathrm{s}}} \\
\times \mathrm{e}^{\mathrm{j} k_{m}(n-1) d \sin \theta}
\end{gathered}
$$

式中: $f$ 表示频率; $S=\left\{r_{\mathrm{s}}, z_{\mathrm{s}}, \theta\right\} ; \Phi$ 表示环境参数 集,包含水深、声速剖面、地声参数等; $\rho\left(z_{\mathrm{s}}\right)$ 表示声 源深度上的介质密度; $M$ 表示传播模态的阶数; $\Psi_{m}(\cdot) 、 k_{m}$ 分别表示第 $m$ 阶模态特征函数 (简称模 态)、对应的水平波数, 他们均由海洋环境和声源频 率共同决定。由于本文主要关注的是参数的不确定 性对检测的影响, 假设频率已知, 因此, 为书写简便, 后文将去掉传递函数对于频率 $f$ 的依赖。

在一定时间范围内, 水下声信道可以看作是一 个线性时不变系统, 结合 (1) 式所示传输函数以及 阵元之间的几何关系, HLA 的接收信号可以在频域 表示为如下形式

$$
\boldsymbol{r}=\boldsymbol{E} \cdot \boldsymbol{a}+\boldsymbol{n}
$$

式中: $\boldsymbol{r}$ 表示 $N \times 1$ 维的接收信号频域快拍; $\boldsymbol{E}$ 为观 测矩阵

$$
\boldsymbol{E}=\left[\begin{array}{ccc}
1 & \cdots & 1 \\
\mathrm{e}^{\mathrm{j} k k_{1} d \sin \theta} & \cdots & \mathrm{e}^{\mathrm{j} k_{M} d \sin \theta} \\
\vdots & & \vdots \\
\mathrm{e}^{\mathrm{j}(N-1) k_{1} d \sin \theta} & \cdots & \mathrm{e}^{\mathrm{j}(N-1) k_{M} d \sin \theta}
\end{array}\right]
$$

可以看出, 水平阵观测矩阵 $\boldsymbol{E}$ 由 $M$ 个平面波方向向 量构成, $\boldsymbol{a}=\left\{\boldsymbol{a}_{m}\right\}_{m=1}^{M}$ 为观测向量, 表示 $M$ 个观测分 量的幅值

$$
a_{m}=s \frac{\mathrm{e}^{\mathrm{j} \pi / 4}}{\rho\left(z_{\mathrm{s}}\right)} \sqrt{\frac{2 \pi}{k_{m} r_{\mathrm{s}}}} \Psi_{m}\left(z_{\mathrm{r}}\right) \Psi_{m}\left(z_{\mathrm{s}}\right) \mathrm{e}^{\mathrm{j} k_{m} r_{\mathrm{s}}}
$$

式中: $s$ 表示辐射声信号的复幅度; $\boldsymbol{n}$ 为 $N \times 1$ 维的背 景噪声向量, 为研究假设, 通常假设其为功率 $2 \sigma^{2}$ 已 知的空间复高斯白噪声, $\boldsymbol{n} \sim C N\left(0,2 \sigma^{2} \boldsymbol{I}_{N}\right), \boldsymbol{I}_{N}$ 为 $N$ 维单位阵。

由 (2) 式所示观测模型可知, 接收到的目标辐 射声信号均可以由观测矩阵中各方向向量线性表 示, 这意味着接收到的目标信号会落人观测矩阵 $\boldsymbol{E}$ 张成的子空间中。

\section{2 不确定环境中水平阵宽容子空间检 测方法}

\section{1 不确定环境中信号子空间的稳健估计}

结合简正波理论和 (1) 式易知, 当环境参数和 
目标位置参数未知时, 各阶水平波数、模态函数、声 源深度、距离、水平方位角均不确定。由 (3) 至 (4) 式可知, 观测向量 $\boldsymbol{a}$ 受各阶模态函数 $\left\{\boldsymbol{\Psi}_{m}(\cdot)\right\}_{m=1}^{M}$ 和 声源深度 $z_{\mathrm{s}}$ 、距离 $r_{\mathrm{s}}$ 的不确定性影响, 观测矩阵 $\boldsymbol{E}$ 受 各阶水平波数 $\left\{k_{m}\right\}_{m=1}^{M}$ 和水平方位角 $\theta$ 的不确定性 影响。

定义中间参量 $\left\{\tilde{k}_{m}\right\}_{m=1}^{M}$

$$
\tilde{k}_{m}=k_{m} \sin \theta
$$

将 (5) 式带人 (3) 式, 将观测矩阵改写为

$$
\boldsymbol{E}=\left[\begin{array}{ccc}
1 & \cdots & 1 \\
\mathrm{e}^{\mathrm{j} \tilde{k}_{1} d} & \cdots & \mathrm{e}^{\mathrm{j} \hat{k}_{M^{d}}} \\
\vdots & & \vdots \\
\mathrm{e}^{\mathrm{j}(N-1) \tilde{\tilde{k}}_{1} d} & \cdots & \mathrm{e}^{\mathrm{j}(N-1) \tilde{k}_{M^{d}}}
\end{array}\right]
$$

结合 (5) 式和 (6) 式可以看出, $\left\{\tilde{k}_{m}\right\}_{m=1}^{M}$ 为一组 不确定的参量, 在观测矩阵中表征由环境参数和目 标位置参数带来的各方向向量的波数不确定性, 称 参量 $\left\{\tilde{k}_{m}\right\}_{m=1}^{M}$ 为不确定波数。显然, 不同组 $\left\{\tilde{k}_{m}\right\}_{m=1}^{M}$ 对应观测矩阵 $\boldsymbol{E}$ 不同, 这意味着不同参数条件下, 接 收到的声源辐射信号会落人不同的子空间中。

观察 (6) 式, 若已知不确定波数 $\left\{\tilde{k}_{m}\right\}_{m=1}^{M}$ 的所有 可能值 $\left\{\tilde{k}_{i}\right\}_{i=1}^{Z}(Z \gg M)$, 利用 $\left\{\tilde{k}_{i}\right\}_{i=1}^{Z}$ 构造形如观测 矩阵 $\boldsymbol{E}$ 的 $N \times Z$ 的矩阵 $\widetilde{\boldsymbol{E}}$

$$
\widetilde{\boldsymbol{E}}=\left[\begin{array}{ccc}
1 & \cdots & 1 \\
\mathrm{e}^{\mathrm{j} \tilde{k}_{1} d} & \cdots & \mathrm{e}^{\mathrm{j} \tilde{k}_{Z} d} \\
\vdots & & \vdots \\
\mathrm{e}^{\mathrm{j}(N-1) \tilde{k}_{1} d} & \cdots & \mathrm{e}^{\mathrm{j}(N-1) \tilde{k}_{Z^{d}}}
\end{array}\right]
$$

此时, 任意参数条件下, $\boldsymbol{E}$ 的所有列向量均可由 $\widetilde{\boldsymbol{E}}$ 的列向量线性表示, 这表明任意不确定参数对应 的观测矩阵 $\boldsymbol{E}$ 张成的子空间均为 $\widetilde{\boldsymbol{E}}$ 张成子空间的子 空间。那么,任意不确定参数对应的接收声源辐射 信号均会落人 $\widetilde{\boldsymbol{E}}$ 张成的子空间中。

为了需要确定 $\left\{\tilde{k}_{i}\right\}_{i=1}^{Z}$, 对不确定波数 $\left\{\tilde{k}_{m}\right\}_{m=1}^{M}$ 的不确定范围进行研究。注意到 1 号简正波对应的 水平波数 $k_{1}$ 大于其余所有的水平波数, 以及正弦函 数 $\sin \theta$ 的取值范围为 $[-1,1]$, 相应的, $\left\{\tilde{k}_{m}\right\}_{m=1}^{M}$ 的 取值范围为

$$
\tilde{k}_{m} \in\left[-k_{1}, k_{1}\right], \quad m=1,2, \cdots, M
$$

进一步, 由于所有简正波对应的水平波数均小 于当前环境最小声速 $c_{\text {min }}$ 对应的波数 $k_{0}=$ $2 \pi f / c_{\text {min }}{ }^{[15]}$, 即

$$
k_{1}<\frac{2 \pi f}{c_{\min }} \triangleq k_{0}
$$

结合 (8) 式和(9)式, 可以得到

$$
\tilde{k}_{m} \in\left(-k_{0}, k_{0}\right), \quad m=1,2, \cdots, M
$$

由 (10) 式可知, $\left\{\tilde{k}_{m}\right\}_{m=1}^{M}$ 不确定范围为 $\left(-k_{0}\right.$, $\left.k_{0}\right)$, 当在该范围内无限精度采样时可以获 $\left\{\tilde{k}_{m}\right\}_{m=1}^{M}$ 的所有可能值 $\left\{\tilde{k}_{i}\right\}_{i=1}^{Z}$ 。 但无限度精度采样显然是 不现实的, 当阵列孔径有限时, 过密的不确定波数采 样会导致矩阵 $\widetilde{\boldsymbol{E}}$ 中各方向向量之间相关性较大,使 得 $\widetilde{\boldsymbol{E}}$ 中存在大量的圥余信息, 这表明可以用恰当的 采样密度代替理想中无限精度的采样。为了研究恰 当的采样密度和剔除 $\widetilde{\boldsymbol{E}}$ 中的圥余信息以提高子空间 方法的性能, 对 $\widetilde{\boldsymbol{E}}$ 进行奇异值分解

$$
\widetilde{\boldsymbol{E}}=\left[\boldsymbol{U}_{s}, \boldsymbol{U}_{n}\right]\left[\begin{array}{ll}
\boldsymbol{\Lambda}_{s} & \\
& \boldsymbol{\Lambda}_{n}
\end{array}\right]\left[\begin{array}{c}
\boldsymbol{V}_{s}^{\mathrm{H}} \\
\boldsymbol{V}_{n}^{\mathrm{H}}
\end{array}\right]
$$

式中: $\boldsymbol{\Lambda}_{s} 、 \boldsymbol{U}_{s} 、 \boldsymbol{V}_{s}$ 分别表示前 $K$ 个较大奇异值对应的 奇异值对角阵、左奇异向量矩阵和右奇异向量矩阵, 为 $\widetilde{\boldsymbol{E}}$ 中主要信息成分; $\boldsymbol{\Lambda}_{n}, \boldsymbol{U}_{n}, \boldsymbol{V}_{n}$ 表示近 0 奇异值对 应的奇异值对角阵、左奇异向量矩阵和右奇异向量 矩阵, 代表冗余信息成分。当不确定波数采样密度 增加, 只增加圥余信息成分而主要信息成分近乎不 变时, 可以认为此时采样密度已经充足, 将圥余信息 成分舍弃。为了保证近似的稳健性, 可以让前 $K$ 个 奇异值和占比尽可能的高。此时, $\widetilde{\boldsymbol{E}}$ 可以由保留的 主要信息成分近似表示为

$$
\widetilde{\boldsymbol{E}} \approx \boldsymbol{U}_{s} \boldsymbol{\Lambda}_{s} \boldsymbol{V}_{s}^{\mathrm{H}}
$$

即 $\widetilde{\boldsymbol{E}}$ 中各方向向量可近似由 $\boldsymbol{U}_{s}$ 中列向量线性表示, 那么 $\boldsymbol{U}_{s}$ 张成的子空间即为得到的信号子空间的稳 健估计,其维数为 $K_{\text {。 }}$

在以往研究中, 通常是通过奇异值的大小来判 断信号子空间的估计误差, 但具有很强的经验性且 物理概念并不清晰, 类比文献 [8], 定义相对投影能 量损失 (relative signal-engergy loss, RSL) 来确定信号 子空间的估计误差。 $l_{\mathrm{REL}}$ 表示接收信号向估计信号 子空间正交投影时的能量损失, 比通过奇异值大小 的方法具有更清晰的物理意义

$$
l_{\mathrm{REL}}=\frac{E_{s}-E_{s, \boldsymbol{P}}}{E_{s}}
$$

式中: 信号能量 $E_{s}=\|\boldsymbol{E} \boldsymbol{a}\|_{2}^{2}$; 投影能量 $E_{s, \boldsymbol{P}}=$ $\|\boldsymbol{P} \cdot \boldsymbol{E} \boldsymbol{a}\|_{2}^{2} ; \boldsymbol{P}$ 为子空间 $W$ 的正交投影矩阵

$$
\boldsymbol{P}=\boldsymbol{U}_{s} \boldsymbol{U}_{s}^{\mathrm{H}}
$$

$\boldsymbol{E} \boldsymbol{a}$ 表示某一个环境参数条件下水平阵接收到 的声源辐射信号。对于某一个已知参数不确定范围 的不确定海洋环境, 如图 2 所示, 当遍历所有可能的 
海洋环境和声源位置组合, 对应 $\boldsymbol{E} \boldsymbol{a}$ 向子空间 $W$ 投影 的 REL 均不超过某一极小值 $\delta$ 时, 表明 $\boldsymbol{U}_{s}$ 张成的子 空间作为信号子空间的估计具有稳健性。在实际应 用中, 当 $\delta<0.01$ 时, 即任意参数下阵列接收声源辐 射信号的 REL 均小于 $1 \%$, 此时可以认为估计的子 空间 $W$ 在所研究环境中信号子空间的稳健估计。

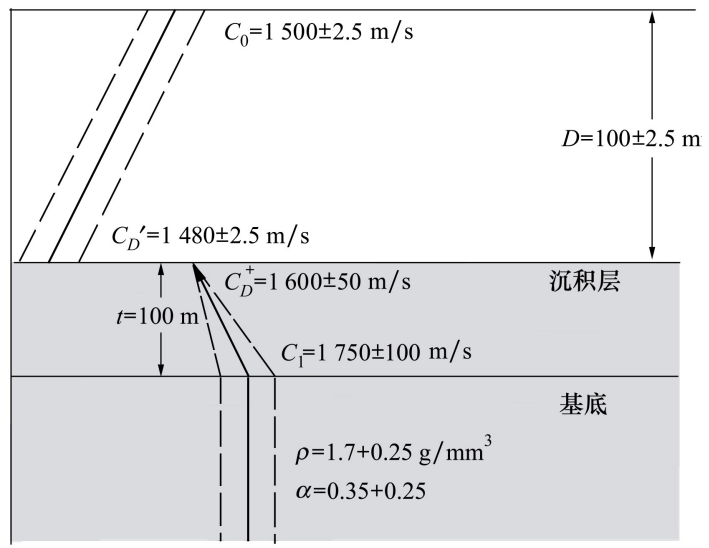

图 2 不确定浅海环境标准失配测试模型

\section{2 稳健子空间检测器}

根据 (2) 式所示水平阵观测模型, 可以将水平 阵信号检测问题建立为如下二元假设检验问题

$$
\begin{gathered}
H_{0}: \boldsymbol{r}=\boldsymbol{n} \\
H_{1}: \boldsymbol{r}=\boldsymbol{E} \boldsymbol{a}+\boldsymbol{n}
\end{gathered}
$$

式中: $H_{0}$ 为 0 假设, 表示只有噪声存在; $H_{1}$ 为备选假 设, 表示有信号存在, 给定接收信号 $\boldsymbol{r}$, 判断他属于 $H_{0}$ 假设还是 $H_{1}$ 假设。

对于 (15) 式所示检验问题的广义似然比检测 器的检验统计量为

$$
T(\boldsymbol{r})=\boldsymbol{r}^{\mathrm{H}} \boldsymbol{E}\left(\boldsymbol{E}^{\mathrm{H}} \boldsymbol{E}\right){ }^{-1} \boldsymbol{E}^{\mathrm{H}} \boldsymbol{r}
$$

式中, $\boldsymbol{E}\left(\boldsymbol{E}^{\mathrm{H}} \boldsymbol{E}\right)^{-1} \boldsymbol{E}^{\mathrm{H}}$ 是 $\boldsymbol{E}$ 张成的信号子空间的正交 投影矩阵。根据之前的分析, 不确定环境中, $\boldsymbol{U}_{s}$ 张 成的子空间为信号子空间的稳健估计, 因此, 结合 (16) 式和(18) 式, $\boldsymbol{U}_{s}$ 张成的子空间的正交投影矩 阵 $\boldsymbol{P}$ 代替 $\boldsymbol{E}\left(\boldsymbol{E}^{\mathrm{H}} \boldsymbol{E}\right)^{-1} \boldsymbol{E}^{\mathrm{H}}$, 得到 HRSD 的检验统计量 为

$$
T_{\mathrm{HRSD}}(\boldsymbol{r})=\boldsymbol{r}^{\mathrm{H}} \boldsymbol{P} \boldsymbol{r}
$$

$T_{\mathrm{HRSD}}(\boldsymbol{r})$ 的统计分布为

$$
\begin{gathered}
T_{\mathrm{HRSD}}(\boldsymbol{r}) \mid H_{1} \sim \chi^{\prime 2}{ }_{2 K}(\beta) \\
T_{\mathrm{HRSD}}(\boldsymbol{r}) \mid H_{0} \sim \chi_{2 K}^{2}
\end{gathered}
$$

式中,非中心化参量 $\beta$ 为

$$
\beta=\frac{E_{s, P}}{\sigma^{2}}
$$

由 (18) 式可知, HRSD 是一个卡方类型检测器。 根据卡方随机变量的统计特性可知, HRSD 的检测 概率随非中心化参量 $\beta$ 增大而增大, 随自由度 $2 K$ 增 大而减小,其中非中心化参量与投影到信号子空间 上的信号能量成正比, 自由度为信号子空间维数 $K$ 的 2 倍。

结合 (13) 式, 将(19) 式改写为

$$
\beta=\left(1-l_{\mathrm{REL}}\right) \frac{E_{s}}{\sigma^{2}}
$$

那么, HRSD 的稳健性可以由 REL 度量, 当各 参数条件下均有 REL $\leqslant 1$ 时, 可将 $(20)$ 式近似表 示为

$$
\beta \approx \frac{E_{s}}{\sigma^{2}}
$$

如(21) 式所示, 此时非中心化参量 $\beta$ 只与接收 信号能量和噪声功率有关, 与环境参数和目标声源 位置参数无关, 这表明 HRSD 在信号子空间估计准 确时具有很高的稳健性。

\section{3 仿真分析}

本节通过在标准不确定浅海波导中进行仿真实 验, 对 HRSD 的有效性进行验证。首先通过等不确 定波数采样估计信号子空间, 仿真信号子空间 REL 验证信号子空间稳健性, 而后, 通过 ROC 曲线和 $P_{d}-R_{\mathrm{SN}}$ 曲线仿真给出 HRSD 检测性能, 并将 HRSD 的性能与 GLRD、ED 进行对比, 来说明 HRSD 的性 能优势。

仿真中使用的标准不确定波导如图 2 所示。他 是由 1993 年美国 NRL 提出的 1 个标准失配模 型 ${ }^{[16]}$,各参数的意义及取值范围在表 1 中给出。

表 1 失配测试模型中参数意义及取值范围

\begin{tabular}{cc}
\hline 参数名 & 取值范围 \\
\hline 海水深度 $D / \mathrm{m}$ & $100 \pm \Delta D$ \\
海面声速 $C_{0} /\left(\mathrm{m} \cdot \mathrm{s}^{-1}\right)$ & $1500 \pm \Delta C_{0}$ \\
海底声速 $C_{D}^{-} /\left(\mathrm{m} \cdot \mathrm{s}^{-1}\right)$ & $1480 \pm \Delta C_{D}^{-}$ \\
沉积层声速 $C_{D}^{+} /\left(\mathrm{m} \cdot \mathrm{s}^{-1}\right)$ & $1600 \pm \Delta C_{D}^{+}$ \\
基底声速 $C_{1} /\left(\mathrm{m} \cdot \mathrm{s}^{-1}\right)$ & $1750 \pm \Delta C_{1}$ \\
底质密度 $\rho /\left(\mathrm{g} \cdot \mathrm{cm}^{-3}\right)$ & $1.7 \pm \Delta \rho$ \\
吸收系数 $\alpha$ & $0.35 \pm \Delta \alpha$ \\
沉积层厚度 $t / \mathrm{m}$ & 100 \\
\hline
\end{tabular}


除环境参数, 由于目标位置也是未知的, 仿真 中, 目标距离 $r_{\mathrm{s}}=6 \pm 4 \mathrm{~km}$, 深度 $z_{\mathrm{s}}=50 \pm 50 \mathrm{~m}$, 水平方 位角 $\theta=0 \pm \pi / 2 \mathrm{rad}$, 环境和目标位置参数在各自取 值范围内均匀分布。

因为本文研究的是低频浅海中的检测问题, 所 以所有仿真过程中计算传输函数都使用 KRAKEN 软件,仿真中假设接收阵均为固定布放在海底的 100 元水平线列阵, 阵列孔径为 $100 \mathrm{~m}$, 声源频率为 $100 \mathrm{~Hz}_{\circ}$

\section{1 信号子空间估计及 REL}

当假设对环境参数分布未知时, 需要恰当地对 声速最小值进行估计, Brekhovskikh 指出, 海洋中声 速的一般范围是 $1450 \sim 1540 \mathrm{~m} / \mathrm{s}^{[17]}$, 结合 (10) 式 可以得到不确定波数 $\left\{\tilde{k}_{m}\right\}_{m=1}^{M}$ 的范围为 $(-0.44$, $0.44)$, 以 0.02 的采样间隔在该范围内均匀取值, 相

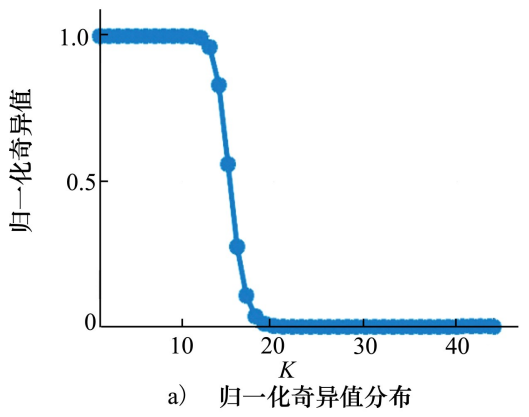

图 $3 \widetilde{\boldsymbol{E}}$ 奇异值分解成会会析

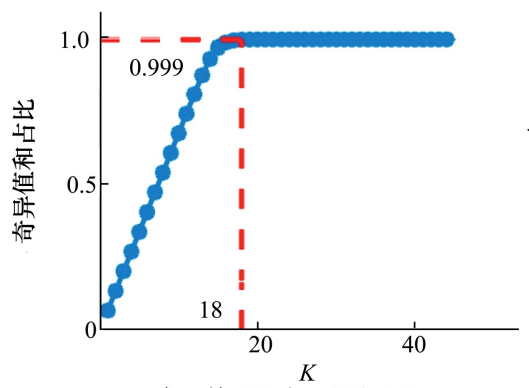

b) 前 $K$ 阶奇异值和占比
应地, 可以得到 44 个波数和对应的重构观测矩阵 $\widetilde{\boldsymbol{E}}_{\circ}$ 对 $\widetilde{\boldsymbol{E}}$ 奇异值分解, 仿真给出相应的归一化奇异 值分布和前 $K$ 阶奇异值之和占奇异值总和比例如 图 3 所示。结合图 3b), 保留前 18 阶奇异值对应的 特征向量用以估计信号子空间,仿真给出 1000 个 随机环境参数实现的 REL 如图 4 所示, 其中每个环 境参数实现中的 REL 是 2000 个不同随机声源位置 处的最大 REL, 由图 4 中可以看出, 1000 个随机环 境参数实现中 REL 在 $10^{-5}$ 量级, 其中 REL 的最大值 为 $9.01 \times 10^{-5}$, 可忽略不计, 这表明当不确定波数采 样间隔为 0.02 , 前 18 阶奇异值对应的特征向量张成 的子空间为信号子空间的稳健估计。

上文给出了采样间隔为 0.02 时的结果, 可以验 论这里不再赘述。 证, 进一步减小采样间隔将导出同样的结果, 相关讨

\subsection{HRSD 性能仿真}

选取不确定波数采样间隔为 0.02 , 固定阵列信 噪比 $\left(R_{\mathrm{SN}}\right)$ 为 $10 \mathrm{~dB}$, 对环境和目标声源位置不确定 参数集 1000 次蒙特卡洛采样, 给出 1000 个不同参 数实现中 HRSD 的 ROC 曲线, 如图 5a) 所示, 其中 灰色曲线对应 1000 个不同参数实现的 ROC 曲线, 蓝色曲线为平均 ROC 曲线。固定虚警概率 $P_{f}=$ 0.1 , 给出 1000 个不同参数实现中 HRSD 的 $P_{d}-R_{\mathrm{SN}}$ 曲线如图 5b) 所示, 同样的灰色曲线对应 1000 个不 同参数实现, 蓝色曲线为平均曲线。由图 5 可以看 出, 1000 次不同参数实现中的 ROC 曲线和 $P_{d}-R_{\mathrm{SN}}$ 曲线与各自的平均曲线非常接近。这一结果表明, 在同一阵列信噪比下, 不同环境参数和目标位置参 数实现中, HRSD 均具有较高的稳健性。将图 5 与 REL 仿真结果对比, 不难看出, HRSD 的稳健性来源 于信号子空间的稳健估计,与理论分析一致。此外， 由图 5b) 可以看出, 当阵列信噪比小于 $0 \mathrm{~dB}$ 时, 此 时 HRSD 的检测概率 $P_{d}$ 趋近虚警概率 $P_{f}$, 各参数

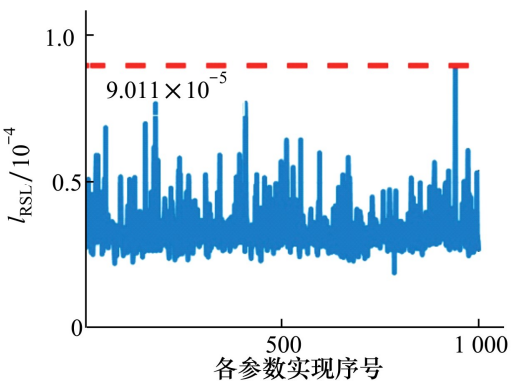

图 4 在 1000 个环境参数实现中信号子空间估计的 REL
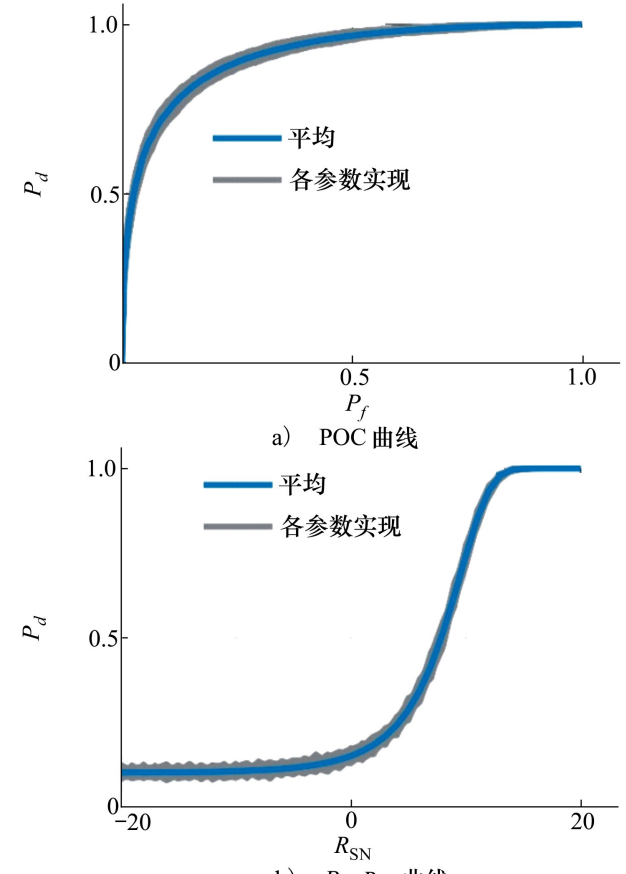

b) $P_{d}-R_{\mathrm{SN}}$ 曲线
图 51000 次参数实现中 HRSD 
实现下的 $P_{d}-R_{\mathrm{SN}}$ 曲线范围增大, HRSD 稳健性下降, 这意味着此时 HRSD 对阵列信噪比有一定的要求, 阵列信噪比太低时检测器失效。

为了更进一步地阐述 HRSD 的检测性能, 需要 将 HRSD 和其他宽容方法做横向对比, 因为在以往 的研究中, MC-GLRD 具有在不确定环境中接近最优 的平均检测性能 ${ }^{[8]}, \mathrm{ED}$ 具有最稳健的检测性能 ${ }^{[9]}$, 所以选取 MC-GLRD 和 ED 作为 HRSD 的对比方法。 首先, 对三者的平均检测性能进行比较。仿真条件 不变, 给出 1000 个不同参数实现中 HRSD、MCGLRD、ED 的平均 ROC 曲线和平均 $P_{d}-R_{\mathrm{SN}}$ 曲线如图 6 所示。在仿真中, MC-GLRD 的不确定参数采样数 为阵元数的 2 倍, 即 $2 N=200$ 。由图 6 中可以看出,
HRSD 的平均检测能力远高于能量检测器, 从图 $6 \mathrm{~b})$ 可以看出, HRSD 在不同信噪比条件下仅略低于 MC-GLRD。这一结果表明了在一定条件下, HRSD 具有较高的检测能力。

接着, 对比 HRSD、MC-GLRD 与 ED 的稳健性。 固定虚警概率 $P_{f}=0.1$, 给出 1000 次参数实现下 MC-GLRD、HRSD、ED 的检测概率 $P_{d}$ 变化如图 7 所 示,可以看出在不同参数条件下 HRSD 具有和 $\mathrm{ED}$ 接近的稳健性,稳健性好于 MC-GLRD。联系 3.1 节 REL 仿真结果表明, 当信号子空间的估计稳健时, HRSD 具有很高的稳健性, 即对环境参数和目标位 置参数的不确定性具有很高的宽容度。

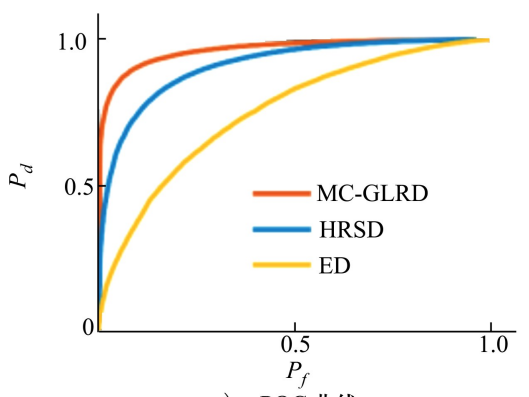

a) POC 曲线

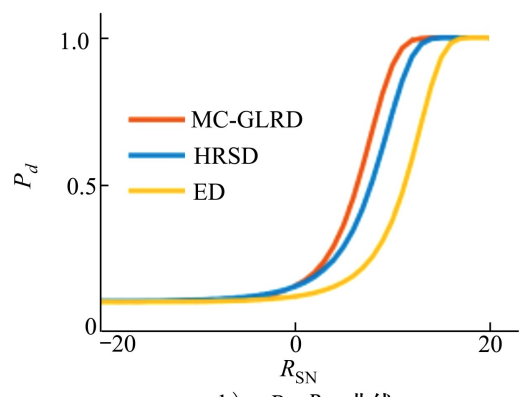

b) $P_{d}-R_{\mathrm{SN}}$ 曲线

图 $6 \mathrm{MC}-\mathrm{GLRD} 、 \mathrm{HRSD} 、 \mathrm{ED}$ 在 1000 次参数实现下平均

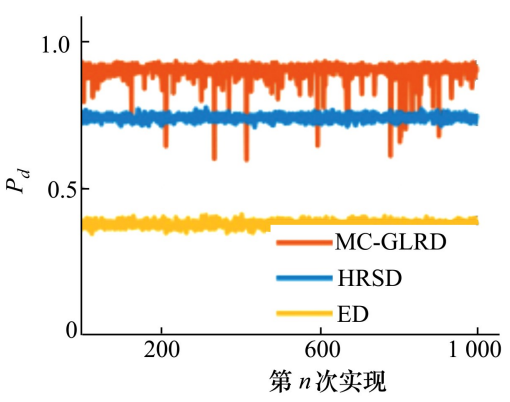

图 7 MC-GLRD、HRSD、ED 在 1000 次 参数实现下检测概率 $P_{d}$ 变化

\section{4 结 论}

本文在水平阵的基础上,结合子空间方法研究 了一种基于稳健子空间的宽容检测器 HRSD, 该检 测器利用了水平阵在不确定特性中的接收特性, 对 于不确定环境中的检测问题有一定的优势。在标准 失配模型条件下, 对 HRSD 进行仿真分析, 在一定条 件下,信号子空间估计的误差可忽略不计, 此时信号
子空间的估计具有稳健性。利用 ROC 曲线、曲线 和不同参数实现中的 变化曲线仿真比较了 HRSD 和 MC-GLRD、ED 的性能差异, HRSD 具有略低于 MC-GLRD 和远高于 ED 的平均检测能力, 同时, HRSD 具有接近 ED 的稳健性, 高于 MC-GLRD。结 合平均检测性能和稳健性, 表明 HRSD 是一种对浅 海不确定环境和未知目标位置参数具有一定宽容性 的高性能检测器。

\section{参考文献:}

[1] SCHMIDT H, BAGGEROER A B, KUPERMAN W A, et al. Environmentally Tolerant Beamforming for High-Resolution Matched Field Processing: Deterministic Mismatch [J]. The Journal of the Acoustical Society of America, 1990, 88(4): 1851-1862

[2] DELBAlzo D R, FEUILLADE C, ROWE M M. Effects of Water-Depth Mismatch on Matched-Field Localization in Shallow Water [J]. The Journal of the Acoustical Society of America, 1988, 83(6) : 2180-2185

[3] 赵航芳, 李建龙, 宫先仪. 不确实海洋中最小方差匹配场波束形成对环境参量失配的灵敏性分析 $[\mathrm{J}]$. 哈尔滨工程大学 
学报, 2011,32(2): 200-208

ZHAO Hangfang, LI Jianlong, GONG Xianyi. Sensitivity of Minimum Variance Matched-Field Beamforming to an Environmental Parameter Mismatch in an Uncertain Ocean Channel[ J]. Journal of Harbin Engineering University, 2011, 32(2) : 200-208 (in Chinese)

[4] 刘宗伟, 孙超, 杜金燕. 不确定海洋声场中的检测性能损失环境敏感度度量 $[\mathrm{J}]$. 物理学报, 2013,62(6): 64303

LIU Zongwei, SUN Chao, DU Jinyan. The Measure of Environmental Sensitivity in Detection Performance Degradation[J]. Acta Physica Sinica, 2013, 62(6): 64303 (in Chinese)

[5] KAY S M. Fundamentals of Statistical Signal Processing[M]. Vpper Sgddle River New Jersey, Prentice Hall PTR, 1993

[6] SHA L, NOLTE L. Effects of Environmental Uncertainties on Sonar Detection Performance Prediction[J]. The Journal of the Acoustical Society of America, 2005,117(1) :1942-1953

[7] LI M, SUN C, WILLETT P. Performance of Mode Space Detector in Uncertain Shallow Water and Its Robust Realization[J]. IEEE Journal of Oceanic Engineering, 2018, 43(1) : 131-144

[8] 刘宗伟, 孙超, 吕连港. 不确定海洋环境中基于蒙特卡罗优化的稳健检测方法 [J]. 声学学报, 2015, 40(5)：665-674 LIU Zongwei, SUN Chao, LYU Liangang. A Robust Signal Detection Method Based on Monte Carlo Optimization in Uncertain Ocean Environment $[\mathrm{J}]$. Acta Acustica, 2015, 40(5) : 665-674 (in Chinese)

[9] SHA L, NOLTE L. Bayesian Sonar Detection Performance Prediction in the Presence of Interference in Uncertain Environments $[\mathrm{J}]$. The Journal of the Acoustical Society of America, 2005,117(4) : 1954-1964

[10] YANG T C. A Method of Range and Depth Estimation by Modal Decomposition[J]. Journal of the Acoustical Society of America, $1987,82(5): 1736-1745$

[11] BOGART C W, Yang T C. Source Localization with Horizontal Arrays in Shallow Water: Spatial Sampling and Effective Aperture [J]. Journal of the Acoustical Society of America, 1994, 96(3):1677-1686

[12] 张同伟, 杨坤德, 马远良, 等. 浅海中水平线列阵深度对匹配场定位性能的影响 $[\mathrm{J}]$. 物理学报, 2010, 59(5):3294-3301 ZHANG Tongwei, YANG Kunde, MA Yuanliang, et al. The Proformance of MFP Localization with a Horizontal Line Array at Different Depths in Shallow Water[J]. Acta Physica Sinica, 2010, 59( 5):3294-3301 (in Chinese)

[13] CONAN E, BONNEL J, NICOLAS B, et al. Using the Trapped Energy Ratio for Source Depth Discrimination with a Horizontal Line Array: Theory and Experimental Results[J]. The Journal of the Acoustical Society of America, 2017, 142( 5) : 2776-2786

[14] SCHARF L L, FRIEDLANDER B. Matched Subspace Detectors [ J]. IEEE Trans on signal processing, 1994,42 ( 8 ): 2146-2157

[15] JENSEN F B, KUPERMAN W A, PORTER M B, et al. Computational Ocean Acoustics[ M]. New York: Springer Science \& Business Media, 2011

[16] PORTER M B . The Matched Field Processing Benchmark Problems [ J ]. Journal of Computational Acoustics, 1994, 2(3): 161-185

[17] BREKHOVSKIKH L M, LYSANOV Y P, BEYER R T. Fundamentals of Ocean Acoustics [ M]. Berlin Heidelberg: SpringorVerlag, 1982 


\title{
A Tolerant Detection Method for Estimating Robust Signal Subspace with Horizontal Arrays in Uncertain Shallow Ocean Environment
}

\author{
WANG Xuan ${ }^{1}$, SUN Chao ${ }^{1}$, XIANG Longfeng ${ }^{2}$, LI Mingyang ${ }^{1}$
}

$\left(\begin{array}{l}\text { 1.School of Marine Science and Technology, Northwestern Polytechnical University, Xi'an 710072, China; } \\ \text { 2.Science and Technology on Electronic Control Laboratory, Chengdu 610036, China }\end{array}\right)$

\begin{abstract}
The environmental parameters are usually uncertain in complex shallow ocean environment and restrict the performance of the matching model-like method. Therefore, we need a more tolerant detection method for detecting underwater targets in the uncertain shallow ocean environment. The previous mode-subspace detection method has the characteristics of both high performance and robustness. However, the robust mode-subspace detector is suitable for vertical arrays and its performance is limited by shallow ocean environment. Therefore, we propose the tolerant detection method for estimating the robust signal subspace with horizontal arrays. We estimate the robust signal subspace by bringing uncertain parameters into the observation matrix of a horizontal array. Combined with the robust signal subspace estimation, we propose a subspace detector that tolerates uncertain parameters. The results on simulation in a uncertain shallow ocean environment show that the detector we proposed has a high average detection capability and a certain tolerance for uncertain parameters.
\end{abstract}

Keywords : tolerant detection; uncertain environment; horizontal array; signal subspace 